<smiles>Cc1ccc(-c2ccc(C(=O)/C=C/c3cccc([N+](=O)[O-])c3)cc2)cc1</smiles>

\title{
Crystal structure of $(E)-1-\left(4^{\prime}\right.$-methyl-[1,1'- biphenyl]-4-yl)-3-(3-nitrophenyl)prop-2- \\ en-1-one
}

T. Vidhyasagar, ${ }^{a}$ K. Rajeswari, ${ }^{a}$ D. Shanthi, ${ }^{a}$

M. Kayalvizhi, ${ }^{b}$ G. Vasuki ${ }^{b}$ and A. Thiruvalluvar ${ }^{\mathrm{c} *}$

a Department of Chemistry, Annamalai University, Annamalai Nagar 608 002, Tamilnadu, India, ${ }^{\mathbf{b}}$ Department of Physics, Kunthavai Naachiar Government Arts College (W) (Autonomous), Thanjavur 613 007, Tamilnadu, India, and ${ }^{\mathrm{C}}$ Postgraduate Research Department of Physics, Rajah Serfoji Government College (Autonomous), Thanjavur 613 005, Tamilnadu, India. *Correspondence e-mail:

thiruvalluvar.a@gmail.com

Received 14 December 2014; accepted 16 December 2014

Edited by H. Stoeckli-Evans, University of Neuchâtel, Switzerland

In the title compound, $\mathrm{C}_{22} \mathrm{H}_{17} \mathrm{NO}_{3}$, the molecule has an $E$ conformation about the $\mathrm{C}=\mathrm{C}$ bond, and the $\mathrm{C}-\mathrm{C}=\mathrm{C}-\mathrm{C}$ torsion angle is $-177.7(3)^{\circ}$. The planes of the terminal benzene rings are twisted by $41.62(16)^{\circ}$, while the biphenyl unit is non-planar, the dihedral angle between the planes of the rings being $38.02(15)^{\circ}$. The dihedral angle between the nitrophenyl ring and the inner benzene ring is $5.29(16)^{\circ}$. In the crystal, molecules are linked by two weak $\mathrm{C}-\mathrm{H} \cdots \pi$ interactions, forming rectangular tubes propagating along the $b$-axis direction.

Keywords: crystal structure; chalcones; $\mathrm{C}-\mathrm{H} \cdots \pi$ interactions.

CCDC reference: 1039539

\section{Related literature}

For the synthesis, antimicrobial, antioxidant activities and growth and characterization of $\pi$-conjugated organic nonlinear optical chalcone derivatives, see: Rajendra Prasad et al. (2008); Lahsasni et al. (2014); Prabhu et al. (2013). For the analysis of Bovine serum albumin in the presence of some phenyl-substituted chalcones, see: Garg et al. (2013). For the crystal structures of related compounds, see: Shanthi et al. (2014); Vidhyasagar et al. (2015).

\subsection{Refinement}

$$
\begin{aligned}
& V=3460.5(3) \AA^{3} \\
& Z=8 \\
& \text { Mo } K \alpha \text { radiation } \\
& \mu=0.09 \mathrm{~mm}^{-1} \\
& T=293 \mathrm{~K} \\
& 0.30 \times 0.20 \times 0.20 \mathrm{~mm}
\end{aligned}
$$

$\beta=103.165(2)^{\circ}$

\subsection{Data collection}

Bruker Kappa APEXII CCD diffractometer

Absorption correction: multi-scan (SADABS; Bruker, 2004) $T_{\min }=0.646, T_{\max }=0.745$

17009 measured reflections 2902 independent reflections 2058 reflections with $I>2 \sigma(I)$ $R_{\text {int }}=0.053$

$R\left[F^{2}>2 \sigma\left(F^{2}\right)\right]=0.067$

$w R\left(F^{2}\right)=0.177$

$S=1.08$

2902 reflections

Table 1

Hydrogen-bond geometry $\left(\AA{ }^{\circ}\right)$.

\author{
236 parameters \\ $\mathrm{H}$-atom parameters constrained \\ $\Delta \rho_{\max }=0.37 \mathrm{e} \AA^{-3}$ \\ $\Delta \rho_{\min }=-0.22 \mathrm{e} \AA^{-3}$
}

$C g 1$ and $C g 3$ are the centroids of rings $\mathrm{C} 1-\mathrm{C} 6$ and $\mathrm{C} 16-\mathrm{C} 21$, respectively

\begin{tabular}{lllll}
\hline$D-\mathrm{H} \cdots A$ & $D-\mathrm{H}$ & $\mathrm{H} \cdots A$ & $D \cdots A$ & $D-\mathrm{H} \cdots A$ \\
\hline $\mathrm{C} 3-\mathrm{H} 3 \cdots C g 3^{\mathrm{i}}$ & 0.93 & 2.99 & $3.531(4)$ & 119 \\
$\mathrm{C} 21-\mathrm{H} 21 \cdots C g 1^{\mathrm{ii}}$ & 0.93 & 2.94 & $3.607(3)$ & 129 \\
\hline
\end{tabular}

Symmetry codes: (i) $-x+\frac{1}{2},-y-\frac{1}{2},-z$; (ii) $-x+\frac{1}{2},-y+\frac{1}{2},-z$.

Data collection: APEX2 (Bruker, 2004); cell refinement: $A P E X 2$ and SAINT (Bruker, 2004); data reduction: SAINT and XPREP (Bruker, 2004); program(s) used to solve structure: SIR2002 (Burla et al., 2003); program(s) used to refine structure: SHELXL2014 (Sheldrick, 2008); molecular graphics: ORTEP-3 for Windows (Farrugia, 2012) and PLATON (Spek, 2009); software used to prepare material for publication: SHELXL2014, PLATON publCIF (Westrip, 2010).

\section{Acknowledgements}

The authors are grateful to the Sophisticated Analytical Instrument Facility (SAIF), IITM, Chennai 600 036, Tamilnadu, India, for the single-crystal X-ray data.

Supporting information for this paper is available from the $\mathrm{IUCr}$ electronic archives (Reference: SU5045). 


\section{References}

Bruker (2004). APEX2, SAINT, XPREP and SADABS. Bruker AXS Inc., Madison, Wisconsin, USA.

Burla, M. C., Camalli, M., Carrozzini, B., Cascarano, G. L., Giacovazzo, C., Polidori, G. \& Spagna, R. (2003). J. Appl. Cryst. 36, 1103.

Farrugia, L. J. (2012). J. Appl. Cryst. 45, 849-854.

Garg, S., Ravish, I. \& Raghav, N. (2013). Int. J. Pharm. Pharm. Sci. 5, 372-375.

Lahsasni, S. A., Al Korbi, F. H. \& Aljaber, N. A. (2014). Chem. Cent. J. doi: $10.1186 / 1752-153 \mathrm{X}-8-32$
Prabhu, A. N., Upadhyaya, V., Jayarama, A. \& Subrahmanya Bhat, K. (2013). Mater. Chem. Phys. 138, 179-185.

Rajendra Prasad, Y., LakshnaRao, A. \& Rambabu, R. (2008). J. Chem. 5, 461466.

Shanthi, D., Vidhya Sagar, T., Kayalvizhi, M., Vasuki, G. \& Thiruvalluvar, A. (2014). Acta Cryst. E70, o809-o810.

Sheldrick, G. M. (2008). Acta Cryst. A64, 112-122.

Spek, A. L. (2009). Acta Cryst. D65, 148-155.

Vidhyasagar, T., Rajeswari, K., Shanthi, D., Kayalvizhi, M., Vasuki, G. \& Thiruvalluvar, A. (2015). Acta Cryst. E71, 1-3.

Westrip, S. P. (2010). J. Appl. Cryst. 43, 920-925. 


\title{
supporting information
}

Acta Cryst. (2015). E71, o65-o66 [https://doi.org/10.1107/S2056989014027443]

\section{Crystal structure of (E)-1-(4'-methyl-[1,1'-biphenyl]-4-yl)-3-(3-nitrophenyl)- prop-2-en-1-one}

\author{
T. Vidhyasagar, K. Rajeswari, D. Shanthi, M. Kayalvizhi, G. Vasuki and A. Thiruvalluvar
}

\section{S1. Comment}

Synthesis and antimicrobial activity of some chalcones derivatives have been reported (Rajendra Prasad et al., 2008). The synthesis, characterization and evaluation of antioxidant activities of some novel chalcone analogues have been reported (Lahsasni et al., 2014). The analysis of Bovine serum albumin in the presence of some phenyl substituted chalcones have been reported (Garg et al., 2013). The growth and characterization of $\pi$ conjugated organic non-linear optical chalcone derivatives were reported (Prabhu et al., 2013). The crystal structures of related compounds were reported (Shanthi et al., 2014; Vidhyasagar et al., 2015). As part of our on-going research on biphenyl chalcone derivatives, the title compound, was synthesized and its crystal structure is reported on herein.

In the title compound, Fig. 1, the molecule exists as an $\mathrm{E}$ conformer with the $\mathrm{C} 5-\mathrm{C} 7-\mathrm{C} 8-\mathrm{C} 9$ torsion angle being $-177.7(3)^{\circ}$. In the molecule, the terminal benzene rings $(\mathrm{C} 1-\mathrm{C} 6$ and $\mathrm{C} 16-\mathrm{C} 21)$ are twisted by an angle of $41.62(16)^{\circ}$, while the biphenyl part $(\mathrm{C} 10-\mathrm{C} 15$ and $\mathrm{C} 16-\mathrm{C} 21)$ is non-planar, the dihedral angle between the rings being $38.02(15)^{\circ}$. The dihedral angle between the nitrophenyl ring $(\mathrm{C} 1-\mathrm{C} 6)$ and the inner phenyl ring $(\mathrm{C} 10-\mathrm{C} 15)$ is $5.29(16)^{\circ}$.

In the crystal, there are two weak $\mathrm{C} 3-\mathrm{H} 3 \cdots \pi$ and $\mathrm{C} 21-\mathrm{H} 21 \cdots \pi$ interactions (Table 1 and Fig. 2) involving the terminal methylbenzene ring $(\mathrm{C} 16-\mathrm{C} 21)$ and the terminal nitrobenzene ring $(\mathrm{C} 1-\mathrm{C} 6)$, respectively. This results in the formation of rectangular tubes propagating along [010]. No classic hydrogen bonds are observed.

\section{S2. Experimental}

A mixture of 4-acetyl-4'-methylbiphenyl (3.43 g, $10 \mathrm{mmol})$ and 3-nitro benzaldehyde (1.07 g, $10 \mathrm{mmol})$ in ethanol (25 $\mathrm{ml})$ in the presence of $\mathrm{NaOH}(10 \mathrm{ml} 30 \%)$ were heated in a water bath for $30 \mathrm{~min}$. and then allowed to cool. The solid that separated was filtered and recrystallized from ethanol. The yellow crystals of the title compound, used for the X-ray diffraction study, were grown by slow evaporation of a solution in acetone (yield: $2.5 \mathrm{~g}, 70 \%$ ).

\section{S3. Refinement}

All $\mathrm{H}$-atoms were positioned geometrically and allowed to ride on their parent atoms, with $\mathrm{C}-\mathrm{H}=0.93-0.96 \AA$ with $U_{\text {iso }}(\mathrm{H})=1.5 U_{\mathrm{eq}}(\mathrm{C})$ for methyl $\mathrm{H}$ atoms and $=1.2 U_{\mathrm{eq}}(\mathrm{C})$ for other $\mathrm{H}$ atoms. 


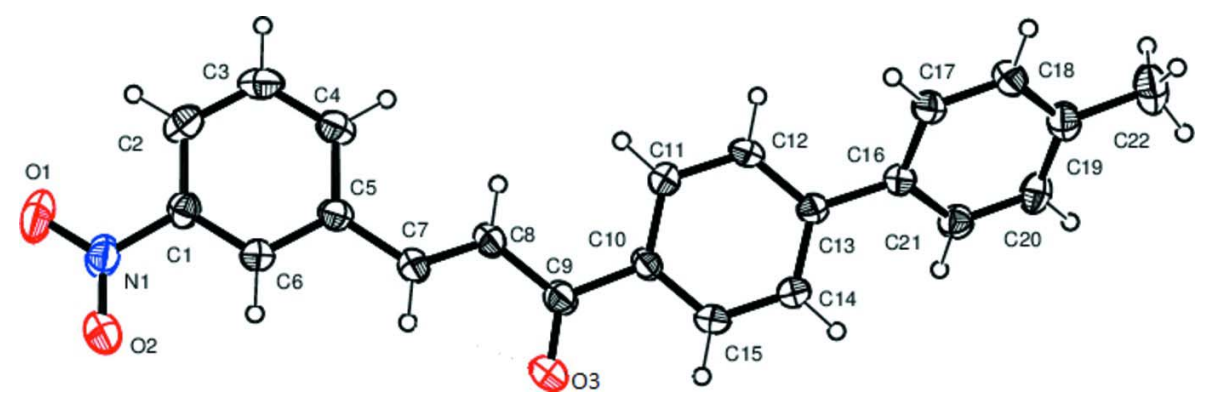

Figure 1

The molecular structure of the title compound, with atom labelling. Displacement ellipsoids are drawn at the $30 \%$ probability level.

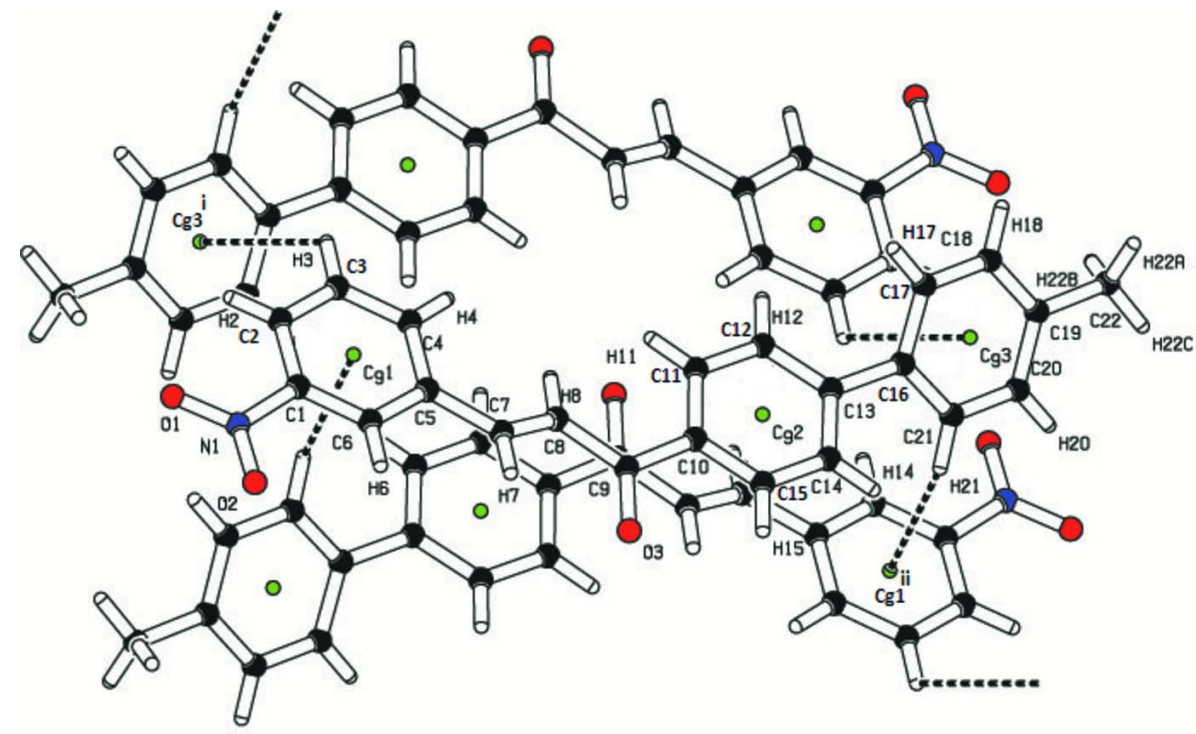

Figure 2

The partial packing of the title compound, showing the two weak $\mathrm{C}-\mathrm{H} \cdots \pi$ interactions (see Table 1 for details).

(E)-1-(4'-Methyl-[1,1'-biphenyl]-4-yl)-3-(3-nitrophenyl)prop-2-en-1-one

\section{Crystal data}

\section{$\mathrm{C}_{22} \mathrm{H}_{17} \mathrm{NO}_{3}$}

$M_{r}=343.37$

Monoclinic, $C 2 / c$

Hall symbol: $-\mathrm{C} 2 \mathrm{yc}$

$a=17.8214$ (10) $\AA$

$b=6.1630(3) \AA$

$c=32.3569$ (19) $\AA$

$\beta=103.165(2)^{\circ}$

$V=3460.5(3) \AA^{3}$

$Z=8$
$F(000)=1440$

$D_{\mathrm{x}}=1.318 \mathrm{Mg} \mathrm{m}^{-3}$

Melting point: $462.3 \mathrm{~K}$

Mo $K \alpha$ radiation, $\lambda=0.71073 \AA$

Cell parameters from 5055 reflections

$\theta=2.4-23.5^{\circ}$

$\mu=0.09 \mathrm{~mm}^{-1}$

$T=293 \mathrm{~K}$

Block, yellow

$0.30 \times 0.20 \times 0.20 \mathrm{~mm}$ 


\section{Data collection}

Bruker Kappa APEXII CCD diffractometer

Radiation source: fine-focus sealed tube Graphite monochromator

$\omega$ and $\varphi$ scan

Absorption correction: multi-scan (SADABS; Bruker, 2004)

$T_{\min }=0.646, T_{\max }=0.745$

\section{Refinement}

Refinement on $F^{2}$

Least-squares matrix: full

$R\left[F^{2}>2 \sigma\left(F^{2}\right)\right]=0.067$

$w R\left(F^{2}\right)=0.177$

$S=1.08$

2902 reflections

236 parameters

0 restraints
17009 measured reflections

2902 independent reflections

2058 reflections with $I>2 \sigma(I)$

$R_{\text {int }}=0.053$

$\theta_{\text {max }}=24.7^{\circ}, \theta_{\min }=2.4^{\circ}$

$h=-20 \rightarrow 20$

$k=-7 \rightarrow 7$

$l=-37 \rightarrow 37$

Hydrogen site location: inferred from neighbouring sites

$\mathrm{H}$-atom parameters constrained

$w=1 /\left[\sigma^{2}\left(F_{\mathrm{o}}^{2}\right)+(0.0622 P)^{2}+6.9426 P\right]$

where $P=\left(F_{\mathrm{o}}^{2}+2 F_{\mathrm{c}}^{2}\right) / 3$

$(\Delta / \sigma)_{\max }<0.001$

$\Delta \rho_{\max }=0.37 \mathrm{e} \AA^{-3}$

$\Delta \rho_{\min }=-0.22$ e $\AA^{-3}$

Special details

Geometry. Bond distances, angles etc. have been calculated using the rounded fractional coordinates. All su's are estimated from the variances of the (full) variance-covariance matrix. The cell e.s.d.'s are taken into account in the estimation of distances, angles and torsion angles

Fractional atomic coordinates and isotropic or equivalent isotropic displacement parameters $\left(\AA^{2}\right)$

\begin{tabular}{lllll}
\hline & $x$ & $y$ & $z$ & $U_{\text {iso }} * / U_{\text {eq }}$ \\
\hline O1 & $0.5576(2)$ & $-0.0419(5)$ & $-0.18992(10)$ & $0.1050(16)$ \\
O2 & $0.57708(17)$ & $0.2525(5)$ & $-0.15451(8)$ & $0.0826(11)$ \\
O3 & $0.39470(16)$ & $0.5129(4)$ & $0.01333(8)$ & $0.0742(10)$ \\
N1 & $0.55103(17)$ & $0.0708(6)$ & $-0.16008(9)$ & $0.0619(11)$ \\
C1 & $0.50961(16)$ & $-0.0197(5)$ & $-0.12946(9)$ & $0.0448(10)$ \\
C2 & $0.48178(19)$ & $-0.2286(6)$ & $-0.13485(11)$ & $0.0571(12)$ \\
C3 & $0.4412(2)$ & $-0.3050(5)$ & $-0.10630(12)$ & $0.0619(14)$ \\
C4 & $0.42788(19)$ & $-0.1752(5)$ & $-0.07404(11)$ & $0.0543(12)$ \\
C5 & $0.45527(18)$ & $0.0367(5)$ & $-0.06918(9)$ & $0.0442(10)$ \\
C6 & $0.49828(17)$ & $0.1111(5)$ & $-0.09729(9)$ & $0.0427(10)$ \\
C7 & $0.43736(19)$ & $0.1877(5)$ & $-0.03729(9)$ & $0.0517(11)$ \\
C8 & $0.38766(19)$ & $0.1586(5)$ & $-0.01312(10)$ & $0.0524(11)$ \\
C9 & $0.37335(18)$ & $0.3262(5)$ & $0.01629(10)$ & $0.0495(11)$ \\
C10 & $0.33265(17)$ & $0.2675(5)$ & $0.05025(9)$ & $0.0411(10)$ \\
C11 & $0.30439(18)$ & $0.0609(5)$ & $0.05492(10)$ & $0.0481(11)$ \\
C12 & $0.26955(18)$ & $0.0165(5)$ & $0.08822(10)$ & $0.0479(11)$ \\
C13 & $0.26254(17)$ & $0.1744(5)$ & $0.11789(9)$ & $0.0398(9)$ \\
C14 & $0.2909(2)$ & $0.3785(5)$ & $0.11259(10)$ & $0.0527(11)$ \\
C15 & $0.32487(19)$ & $0.4245(5)$ & $0.07947(10)$ & $0.0525(11)$ \\
C16 & $0.22680(17)$ & $0.1252(5)$ & $0.15388(9)$ & $0.0411(10)$ \\
C17 & $0.23877(18)$ & $-0.0738(5)$ & $0.17499(10)$ & $0.0484(11)$ \\
C18 & $0.20358(19)$ & $-0.1232(5)$ & $0.20751(10)$ & $0.0527(11)$ \\
& & & &
\end{tabular}




$\begin{array}{lllll}\text { C19 } & 0.15524(19) & 0.0243(6) & 0.22083(10) & 0.0529(11) \\ \text { C20 } & 0.14488(18) & 0.2233(6) & 0.20100(10) & 0.0542(11) \\ \text { C21 } & 0.17998(18) & 0.2742(5) & 0.16818(10) & 0.0491(1) \\ \text { C22 } & 0.1162(2) & -0.0328(8) & 0.25609(12) & 0.0813(18) \\ \text { H2 } & 0.49001 & -0.31504 & -0.15695 & 0.0685^{*} \\ \text { H3 } & 0.42262 & -0.44648 & -0.10885 & 0.0742^{*} \\ \text { H4 } & 0.40014 & -0.23004 & -0.05526 & 0.0652^{*} \\ \text { H6 } & 0.51930 & 0.24991 & -0.09416 & 0.0512^{*} \\ \text { H7 } & 0.46418 & 0.31838 & -0.03384 & 0.0616^{*} \\ \text { H8 } & 0.36098 & 0.02805 & -0.01481 & 0.068^{*} \\ \text { H11 } & 0.30885 & -0.04782 & 0.03569 & 0.0576^{*} \\ \text { H12 } & 0.25041 & -0.12199 & 0.09077 & 0.0572^{*} \\ \text { H14 } & 0.28687 & 0.48747 & 0.13186 & 0.0631^{*} \\ \text { H15 } & 0.34298 & 0.56398 & 0.07669 & 0.0626^{*} \\ \text { H17 } & 0.27127 & -0.17533 & 0.16688 & 0.0578^{*} \\ \text { H18 } & 0.21237 & -0.25769 & 0.22078 & 0.0632^{*} \\ \text { H20 } & 0.11360 & 0.32586 & 0.20984 & 0.0650^{*} \\ \text { H21 } & 0.17207 & 0.41032 & 0.15553 & 0.0588^{*} \\ \text { H22A } & 0.15172 & -0.10892 & 0.27806 & 0.1225^{*} \\ \text { H22B } & 0.07234 & -0.12346 & 0.24514 & 0.1225^{*} \\ \text { H22C } & 0.09953 & 0.09766 & 0.26755 & 0.1225^{*}\end{array}$

Atomic displacement parameters $\left(\AA^{2}\right)$

\begin{tabular}{lllllll}
\hline & $U^{11}$ & $U^{22}$ & $U^{33}$ & $U^{12}$ & $U^{13}$ & $U^{23}$ \\
\hline O1 & $0.131(3)$ & $0.115(3)$ & $0.090(2)$ & $-0.020(2)$ & $0.069(2)$ & $-0.045(2)$ \\
O2 & $0.102(2)$ & $0.080(2)$ & $0.0823(19)$ & $-0.0289(17)$ & $0.0551(17)$ & $-0.0140(16)$ \\
O3 & $0.104(2)$ & $0.0562(16)$ & $0.0751(17)$ & $-0.0230(15)$ & $0.0470(16)$ & $-0.0017(13)$ \\
N1 & $0.0566(18)$ & $0.072(2)$ & $0.063(2)$ & $-0.0032(16)$ & $0.0260(15)$ & $-0.0152(17)$ \\
C1 & $0.0358(16)$ & $0.051(2)$ & $0.0489(18)$ & $0.0039(15)$ & $0.0121(14)$ & $-0.0019(15)$ \\
C2 & $0.052(2)$ & $0.051(2)$ & $0.067(2)$ & $0.0034(17)$ & $0.0106(17)$ & $-0.0158(18)$ \\
C3 & $0.062(2)$ & $0.0386(19)$ & $0.083(3)$ & $-0.0083(17)$ & $0.012(2)$ & $-0.0014(18)$ \\
C4 & $0.056(2)$ & $0.049(2)$ & $0.058(2)$ & $-0.0107(16)$ & $0.0130(16)$ & $0.0048(16)$ \\
C5 & $0.0493(18)$ & $0.0413(18)$ & $0.0405(16)$ & $-0.0064(15)$ & $0.0069(14)$ & $0.0018(14)$ \\
C6 & $0.0444(17)$ & $0.0391(17)$ & $0.0449(17)$ & $-0.0019(14)$ & $0.0109(14)$ & $-0.0005(14)$ \\
C7 & $0.064(2)$ & $0.050(2)$ & $0.0460(18)$ & $-0.0110(16)$ & $0.0227(16)$ & $-0.0018(15)$ \\
C8 & $0.060(2)$ & $0.053(2)$ & $0.0498(19)$ & $-0.0167(17)$ & $0.0244(17)$ & $-0.0032(16)$ \\
C9 & $0.053(2)$ & $0.052(2)$ & $0.0444(18)$ & $-0.0119(16)$ & $0.0133(15)$ & $-0.0002(15)$ \\
C10 & $0.0451(17)$ & $0.0422(18)$ & $0.0381(16)$ & $-0.0045(14)$ & $0.0139(13)$ & $0.0057(14)$ \\
C11 & $0.058(2)$ & $0.0442(18)$ & $0.0455(18)$ & $-0.0050(16)$ & $0.0189(16)$ & $-0.0059(14)$ \\
C12 & $0.057(2)$ & $0.0345(17)$ & $0.056(2)$ & $-0.0096(15)$ & $0.0207(16)$ & $0.0027(14)$ \\
C13 & $0.0437(17)$ & $0.0346(16)$ & $0.0430(16)$ & $0.0010(13)$ & $0.0136(14)$ & $0.0000(13)$ \\
C14 & $0.074(2)$ & $0.0369(18)$ & $0.0525(19)$ & $-0.0066(17)$ & $0.0257(18)$ & $-0.0051(15)$ \\
C15 & $0.069(2)$ & $0.0367(18)$ & $0.055(2)$ & $-0.0110(16)$ & $0.0211(17)$ & $-0.0010(15)$ \\
C16 & $0.0449(18)$ & $0.0383(17)$ & $0.0405(16)$ & $-0.0025(14)$ & $0.0109(14)$ & $-0.0005(13)$ \\
C17 & $0.055(2)$ & $0.0412(18)$ & $0.0533(19)$ & $0.0002(15)$ & $0.0214(16)$ & $0.0005(15)$ \\
C18 & $0.063(2)$ & $0.050(2)$ & $0.0464(19)$ & $-0.0043(17)$ & $0.0155(17)$ & $0.0082(15)$ \\
C19 & $0.051(2)$ & $0.064(2)$ & $0.0443(18)$ & $-0.0056(17)$ & $0.0122(15)$ & $-0.0019(17)$ \\
& & & & & &
\end{tabular}




\begin{tabular}{lllllll}
$\mathrm{C} 20$ & $0.052(2)$ & $0.065(2)$ & $0.0488(19)$ & $0.0109(17)$ & $0.0180(16)$ & $-0.0065(17)$ \\
$\mathrm{C} 21$ & $0.0530(19)$ & $0.0433(19)$ & $0.0508(18)$ & $0.0059(16)$ & $0.0117(16)$ & $0.0006(15)$ \\
$\mathrm{C} 22$ & $0.084(3)$ & $0.110(4)$ & $0.059(2)$ & $-0.005(3)$ & $0.035(2)$ & $0.002(2)$ \\
\hline
\end{tabular}

Geometric parameters $\left(\AA,{ }^{\circ}\right)$

\begin{tabular}{|c|c|c|c|}
\hline $\mathrm{O} 1-\mathrm{N} 1$ & $1.217(4)$ & $\mathrm{C} 17-\mathrm{C} 18$ & $1.376(5)$ \\
\hline $\mathrm{O} 2-\mathrm{N} 1$ & $1.210(5)$ & $\mathrm{C} 18-\mathrm{C} 19$ & $1.387(5)$ \\
\hline $\mathrm{O} 3-\mathrm{C} 9$ & $1.223(4)$ & $\mathrm{C} 19-\mathrm{C} 20$ & $1.377(5)$ \\
\hline $\mathrm{N} 1-\mathrm{C} 1$ & $1.473(4)$ & $\mathrm{C} 19-\mathrm{C} 22$ & $1.507(5)$ \\
\hline $\mathrm{C} 1-\mathrm{C} 2$ & $1.376(5)$ & $\mathrm{C} 20-\mathrm{C} 21$ & $1.386(5)$ \\
\hline $\mathrm{C} 1-\mathrm{C} 6$ & $1.367(4)$ & $\mathrm{C} 2-\mathrm{H} 2$ & 0.9300 \\
\hline $\mathrm{C} 2-\mathrm{C} 3$ & $1.379(5)$ & $\mathrm{C} 3-\mathrm{H} 3$ & 0.9300 \\
\hline $\mathrm{C} 3-\mathrm{C} 4$ & $1.378(5)$ & $\mathrm{C} 4-\mathrm{H} 4$ & 0.9300 \\
\hline $\mathrm{C} 4-\mathrm{C} 5$ & $1.391(4)$ & $\mathrm{C} 6-\mathrm{H} 6$ & 0.9300 \\
\hline $\mathrm{C} 5-\mathrm{C} 6$ & $1.394(4)$ & $\mathrm{C} 7-\mathrm{H} 7$ & 0.9300 \\
\hline $\mathrm{C} 5-\mathrm{C} 7$ & $1.477(4)$ & $\mathrm{C} 8-\mathrm{H} 8$ & 0.9300 \\
\hline $\mathrm{C} 7-\mathrm{C} 8$ & $1.321(5)$ & C11-H11 & 0.9300 \\
\hline $\mathrm{C} 8-\mathrm{C} 9$ & $1.466(4)$ & $\mathrm{C} 12-\mathrm{H} 12$ & 0.9300 \\
\hline $\mathrm{C} 9-\mathrm{C} 10$ & $1.493(4)$ & C14-H14 & 0.9300 \\
\hline $\mathrm{C} 10-\mathrm{C} 11$ & $1.390(4)$ & C15-H15 & 0.9300 \\
\hline $\mathrm{C} 10-\mathrm{C} 15$ & $1.382(4)$ & C17-H17 & 0.9300 \\
\hline $\mathrm{C} 11-\mathrm{C} 12$ & $1.388(5)$ & C18-H18 & 0.9300 \\
\hline $\mathrm{C} 12-\mathrm{C} 13$ & $1.392(4)$ & $\mathrm{C} 20-\mathrm{H} 20$ & 0.9300 \\
\hline $\mathrm{C} 13-\mathrm{C} 14$ & $1.381(4)$ & $\mathrm{C} 21-\mathrm{H} 21$ & 0.9300 \\
\hline $\mathrm{C} 13-\mathrm{C} 16$ & $1.480(4)$ & $\mathrm{C} 22-\mathrm{H} 22 \mathrm{~A}$ & 0.9600 \\
\hline $\mathrm{C} 14-\mathrm{C} 15$ & $1.375(5)$ & $\mathrm{C} 22-\mathrm{H} 22 \mathrm{~B}$ & 0.9600 \\
\hline $\mathrm{C} 16-\mathrm{C} 17$ & $1.396(4)$ & $\mathrm{C} 22-\mathrm{H} 22 \mathrm{C}$ & 0.9600 \\
\hline $\mathrm{C} 16-\mathrm{C} 21$ & $1.389(4)$ & & \\
\hline $\mathrm{O} 1-\mathrm{N} 1-\mathrm{O} 2$ & $122.9(3)$ & $\mathrm{C} 19-\mathrm{C} 20-\mathrm{C} 21$ & $121.5(3)$ \\
\hline $\mathrm{O} 1-\mathrm{N} 1-\mathrm{C} 1$ & $118.1(3)$ & $\mathrm{C} 16-\mathrm{C} 21-\mathrm{C} 20$ & $121.1(3)$ \\
\hline $\mathrm{O} 2-\mathrm{N} 1-\mathrm{C} 1$ & $119.0(3)$ & $\mathrm{C} 1-\mathrm{C} 2-\mathrm{H} 2$ & 121.00 \\
\hline $\mathrm{N} 1-\mathrm{C} 1-\mathrm{C} 2$ & $119.4(3)$ & $\mathrm{C} 3-\mathrm{C} 2-\mathrm{H} 2$ & 121.00 \\
\hline $\mathrm{N} 1-\mathrm{C} 1-\mathrm{C} 6$ & $118.2(3)$ & $\mathrm{C} 2-\mathrm{C} 3-\mathrm{H} 3$ & 119.00 \\
\hline $\mathrm{C} 2-\mathrm{C} 1-\mathrm{C} 6$ & $122.4(3)$ & $\mathrm{C} 4-\mathrm{C} 3-\mathrm{H} 3$ & 119.00 \\
\hline $\mathrm{C} 1-\mathrm{C} 2-\mathrm{C} 3$ & $117.6(3)$ & $\mathrm{C} 3-\mathrm{C} 4-\mathrm{H} 4$ & 120.00 \\
\hline $\mathrm{C} 2-\mathrm{C} 3-\mathrm{C} 4$ & $121.1(3)$ & $\mathrm{C} 5-\mathrm{C} 4-\mathrm{H} 4$ & 120.00 \\
\hline $\mathrm{C} 3-\mathrm{C} 4-\mathrm{C} 5$ & $121.0(3)$ & $\mathrm{C} 1-\mathrm{C} 6-\mathrm{H} 6$ & 120.00 \\
\hline $\mathrm{C} 4-\mathrm{C} 5-\mathrm{C} 6$ & $117.8(3)$ & $\mathrm{C} 5-\mathrm{C} 6-\mathrm{H} 6$ & 120.00 \\
\hline $\mathrm{C} 4-\mathrm{C} 5-\mathrm{C} 7$ & $123.0(3)$ & $\mathrm{C} 5-\mathrm{C} 7-\mathrm{H} 7$ & 116.00 \\
\hline $\mathrm{C} 6-\mathrm{C} 5-\mathrm{C} 7$ & $119.1(3)$ & $\mathrm{C} 8-\mathrm{C} 7-\mathrm{H} 7$ & 116.00 \\
\hline $\mathrm{C} 1-\mathrm{C} 6-\mathrm{C} 5$ & $120.1(3)$ & $\mathrm{C} 7-\mathrm{C} 8-\mathrm{H} 8$ & 119.00 \\
\hline $\mathrm{C} 5-\mathrm{C} 7-\mathrm{C} 8$ & $127.5(3)$ & $\mathrm{C} 9-\mathrm{C} 8-\mathrm{H} 8$ & 119.00 \\
\hline $\mathrm{C} 7-\mathrm{C} 8-\mathrm{C} 9$ & $121.9(3)$ & $\mathrm{C} 10-\mathrm{C} 11-\mathrm{H} 11$ & 120.00 \\
\hline $\mathrm{O} 3-\mathrm{C} 9-\mathrm{C} 8$ & $120.6(3)$ & $\mathrm{C} 12-\mathrm{C} 11-\mathrm{H} 11$ & 120.00 \\
\hline $\mathrm{O} 3-\mathrm{C} 9-\mathrm{C} 10$ & $119.9(3)$ & $\mathrm{C} 11-\mathrm{C} 12-\mathrm{H} 12$ & 119.00 \\
\hline $\mathrm{C} 8-\mathrm{C} 9-\mathrm{C} 10$ & $119.5(3)$ & $\mathrm{C} 13-\mathrm{C} 12-\mathrm{H} 12$ & 119.00 \\
\hline
\end{tabular}




\begin{tabular}{|c|c|}
\hline $\mathrm{C} 9-\mathrm{C} 10-\mathrm{C} 11$ & $123.4(3)$ \\
\hline $\mathrm{C} 9-\mathrm{C} 10-\mathrm{C} 15$ & $118.4(3)$ \\
\hline $\mathrm{C} 11-\mathrm{C} 10-\mathrm{C} 15$ & $118.2(3)$ \\
\hline $\mathrm{C} 10-\mathrm{C} 11-\mathrm{C} 12$ & $120.2(3)$ \\
\hline $\mathrm{C} 11-\mathrm{C} 12-\mathrm{C} 13$ & $121.6(3)$ \\
\hline $\mathrm{C} 12-\mathrm{C} 13-\mathrm{C} 14$ & $117.3(3)$ \\
\hline $\mathrm{C} 12-\mathrm{C} 13-\mathrm{C} 16$ & $121.4(3)$ \\
\hline $\mathrm{C} 14-\mathrm{C} 13-\mathrm{C} 16$ & $121.3(3)$ \\
\hline $\mathrm{C} 13-\mathrm{C} 14-\mathrm{C} 15$ & $121.6(3)$ \\
\hline $\mathrm{C} 10-\mathrm{C} 15-\mathrm{C} 14$ & $121.3(3)$ \\
\hline $\mathrm{C} 13-\mathrm{C} 16-\mathrm{C} 17$ & $121.3(3)$ \\
\hline $\mathrm{C} 13-\mathrm{C} 16-\mathrm{C} 21$ & $121.7(3)$ \\
\hline $\mathrm{C} 17-\mathrm{C} 16-\mathrm{C} 21$ & $117.0(3)$ \\
\hline $\mathrm{C} 16-\mathrm{C} 17-\mathrm{C} 18$ & $121.5(3)$ \\
\hline $\mathrm{C} 17-\mathrm{C} 18-\mathrm{C} 19$ & $121.1(3)$ \\
\hline $\mathrm{C} 18-\mathrm{C} 19-\mathrm{C} 20$ & $117.8(3)$ \\
\hline $\mathrm{C} 18-\mathrm{C} 19-\mathrm{C} 22$ & $120.6(3)$ \\
\hline $\mathrm{C} 20-\mathrm{C} 19-\mathrm{C} 22$ & $121.7(3)$ \\
\hline $\mathrm{O} 1-\mathrm{N} 1-\mathrm{C} 1-\mathrm{C} 2$ & $1.7(5)$ \\
\hline $\mathrm{O} 1-\mathrm{N} 1-\mathrm{C} 1-\mathrm{C} 6$ & $-176.3(3)$ \\
\hline $\mathrm{O} 2-\mathrm{N} 1-\mathrm{C} 1-\mathrm{C} 2$ & $-178.1(3)$ \\
\hline $\mathrm{O} 2-\mathrm{N} 1-\mathrm{C} 1-\mathrm{C} 6$ & $3.9(5)$ \\
\hline $\mathrm{N} 1-\mathrm{C} 1-\mathrm{C} 2-\mathrm{C} 3$ & $-178.0(3)$ \\
\hline $\mathrm{C} 6-\mathrm{C} 1-\mathrm{C} 2-\mathrm{C} 3$ & $0.0(5)$ \\
\hline $\mathrm{N} 1-\mathrm{C} 1-\mathrm{C} 6-\mathrm{C} 5$ & $176.0(3)$ \\
\hline $\mathrm{C} 2-\mathrm{C} 1-\mathrm{C} 6-\mathrm{C} 5$ & $-1.9(5)$ \\
\hline $\mathrm{C} 1-\mathrm{C} 2-\mathrm{C} 3-\mathrm{C} 4$ & $1.2(5)$ \\
\hline $\mathrm{C} 2-\mathrm{C} 3-\mathrm{C} 4-\mathrm{C} 5$ & $-0.3(5)$ \\
\hline $\mathrm{C} 3-\mathrm{C} 4-\mathrm{C} 5-\mathrm{C} 6$ & $-1.7(5)$ \\
\hline $\mathrm{C} 3-\mathrm{C} 4-\mathrm{C} 5-\mathrm{C} 7$ & $175.0(3)$ \\
\hline $\mathrm{C} 4-\mathrm{C} 5-\mathrm{C} 6-\mathrm{C} 1$ & $2.7(5)$ \\
\hline $\mathrm{C} 7-\mathrm{C} 5-\mathrm{C} 6-\mathrm{C} 1$ & $-174.1(3)$ \\
\hline $\mathrm{C} 4-\mathrm{C} 5-\mathrm{C} 7-\mathrm{C} 8$ & $-8.8(5)$ \\
\hline $\mathrm{C} 6-\mathrm{C} 5-\mathrm{C} 7-\mathrm{C} 8$ & $167.9(3)$ \\
\hline $\mathrm{C} 5-\mathrm{C} 7-\mathrm{C} 8-\mathrm{C} 9$ & $-177.7(3)$ \\
\hline $\mathrm{C} 7-\mathrm{C} 8-\mathrm{C} 9-\mathrm{O} 3$ & $15.0(5)$ \\
\hline $\mathrm{C} 7-\mathrm{C} 8-\mathrm{C} 9-\mathrm{C} 10$ & $-164.7(3)$ \\
\hline $\mathrm{O} 3-\mathrm{C} 9-\mathrm{C} 10-\mathrm{C} 11$ & $177.9(3)$ \\
\hline $\mathrm{O} 3-\mathrm{C} 9-\mathrm{C} 10-\mathrm{C} 15$ & $-4.4(5)$ \\
\hline $\mathrm{C} 8-\mathrm{C} 9-\mathrm{C} 10-\mathrm{C} 11$ & $-2.4(5)$ \\
\hline $\mathrm{C} 8-\mathrm{C} 9-\mathrm{C} 10-\mathrm{C} 15$ & $175.3(3)$ \\
\hline $\mathrm{C} 9-\mathrm{C} 10-\mathrm{C} 11-\mathrm{C} 12$ & $177.7(3)$ \\
\hline
\end{tabular}

$\begin{array}{ll}\mathrm{C} 13-\mathrm{C} 14-\mathrm{H} 14 & 119.00 \\ \mathrm{C} 15-\mathrm{C} 14-\mathrm{H} 14 & 119.00 \\ \mathrm{C} 10-\mathrm{C} 15-\mathrm{H} 15 & 119.00 \\ \mathrm{C} 14-\mathrm{C} 15-\mathrm{H} 15 & 119.00 \\ \mathrm{C} 16-\mathrm{C} 17-\mathrm{H} 17 & 119.00 \\ \mathrm{C} 18-\mathrm{C} 17-\mathrm{H} 17 & 119.00 \\ \mathrm{C} 17-\mathrm{C} 18-\mathrm{H} 18 & 119.00 \\ \mathrm{C} 19-\mathrm{C} 18-\mathrm{H} 18 & 119.00 \\ \mathrm{C} 19-\mathrm{C} 20-\mathrm{H} 20 & 119.00 \\ \mathrm{C} 21-\mathrm{C} 20-\mathrm{H} 20 & 119.00 \\ \mathrm{C} 16-\mathrm{C} 21-\mathrm{H} 21 & 119.00 \\ \mathrm{C} 20-\mathrm{C} 21-\mathrm{H} 21 & 119.00 \\ \mathrm{C} 19-\mathrm{C} 22-\mathrm{H} 22 \mathrm{~A} & 110.00 \\ \mathrm{C} 19-\mathrm{C} 22-\mathrm{H} 22 \mathrm{~B} & 110.00 \\ \mathrm{C} 19-\mathrm{C} 22-\mathrm{H} 22 \mathrm{C} & 109.00 \\ \mathrm{H} 22 \mathrm{~A}-\mathrm{C} 22-\mathrm{H} 22 \mathrm{~B} & 109.00 \\ \mathrm{H} 22 \mathrm{~A}-\mathrm{C} 22-\mathrm{H} 22 \mathrm{C} & 109.00 \\ \mathrm{H} 22 \mathrm{~B}-\mathrm{C} 22-\mathrm{H} 22 \mathrm{C} & 109.00\end{array}$

$\mathrm{C} 15-\mathrm{C} 10-\mathrm{C} 11-\mathrm{C} 12 \quad 0.0(5)$

$\mathrm{C} 9-\mathrm{C} 10-\mathrm{C} 15-\mathrm{C} 14 \quad-177.2$ (3)

$\mathrm{C} 11-\mathrm{C} 10-\mathrm{C} 15-\mathrm{C} 14 \quad 0.7(5)$

$\mathrm{C} 10-\mathrm{C} 11-\mathrm{C} 12-\mathrm{C} 13 \quad-0.7(5)$

$\mathrm{C} 11-\mathrm{C} 12-\mathrm{C} 13-\mathrm{C} 14 \quad 0.8$ (5)

$\mathrm{C} 11-\mathrm{C} 12-\mathrm{C} 13-\mathrm{C} 16 \quad-178.7$ (3)

$\mathrm{C} 12-\mathrm{C} 13-\mathrm{C} 14-\mathrm{C} 15 \quad-0.1(5)$

$\mathrm{C} 16-\mathrm{C} 13-\mathrm{C} 14-\mathrm{C} 15 \quad 179.3$ (3)

$\mathrm{C} 12-\mathrm{C} 13-\mathrm{C} 16-\mathrm{C} 17 \quad 37.9$ (4)

$\mathrm{C} 12-\mathrm{C} 13-\mathrm{C} 16-\mathrm{C} 21 \quad-142.1(3)$

$\mathrm{C} 14-\mathrm{C} 13-\mathrm{C} 16-\mathrm{C} 17 \quad-141.5$ (3)

$\mathrm{C} 14-\mathrm{C} 13-\mathrm{C} 16-\mathrm{C} 21 \quad 38.5(5)$

$\mathrm{C} 13-\mathrm{C} 14-\mathrm{C} 15-\mathrm{C} 10 \quad-0.6(5)$

$\mathrm{C} 13-\mathrm{C} 16-\mathrm{C} 17-\mathrm{C} 18 \quad-177.7$ (3)

$\mathrm{C} 21-\mathrm{C} 16-\mathrm{C} 17-\mathrm{C} 18 \quad 2.3(5)$

$\mathrm{C} 13-\mathrm{C} 16-\mathrm{C} 21-\mathrm{C} 20 \quad 177.9$ (3)

$\mathrm{C} 17-\mathrm{C} 16-\mathrm{C} 21-\mathrm{C} 20 \quad-2.1$ (5)

$\mathrm{C} 16-\mathrm{C} 17-\mathrm{C} 18-\mathrm{C} 19 \quad-0.6(5)$

$\mathrm{C} 17-\mathrm{C} 18-\mathrm{C} 19-\mathrm{C} 20 \quad-1.3(5)$

$\mathrm{C} 17-\mathrm{C} 18-\mathrm{C} 19-\mathrm{C} 22 \quad 179.2(3)$

$\mathrm{C} 18-\mathrm{C} 19-\mathrm{C} 20-\mathrm{C} 21 \quad 1.4(5)$

$\mathrm{C} 22-\mathrm{C} 19-\mathrm{C} 20-\mathrm{C} 21 \quad-179.1$ (3)

$\mathrm{C} 19-\mathrm{C} 20-\mathrm{C} 21-\mathrm{C} 16 \quad 0.3(5)$ 
Hydrogen-bond geometry $\left(A,{ }^{\circ}\right)$

$\mathrm{Cg} 1$ and $\mathrm{Cg} 3$ are the centroids of rings $\mathrm{C} 1-\mathrm{C} 6$ and $\mathrm{C} 16-\mathrm{C} 21$, respectively.

\begin{tabular}{lllll}
\hline$D-\mathrm{H} \cdots A$ & $D-\mathrm{H}$ & $\mathrm{H} \cdots A$ & $D \cdots A$ & $D-\mathrm{H} \cdots A$ \\
\hline $\mathrm{C} 3-\mathrm{H} 3 \cdots C g 3^{\mathrm{i}}$ & 0.93 & 2.99 & $3.531(4)$ & 119 \\
$\mathrm{C} 21-\mathrm{H} 21 \cdots C g 1^{\mathrm{ii}}$ & 0.93 & 2.94 & $3.607(3)$ & 129 \\
\hline
\end{tabular}

Symmetry codes: (i) $-x+1 / 2,-y-1 / 2,-z$; (ii) $-x+1 / 2,-y+1 / 2,-z$. 